\title{
Identification by Genetic Algorithm of a Constitutive Law Taking into Account the Effects of Hydrostatic Pressure and Speeds
}

\author{
J.C. Grandidier ${ }^{1}$ and É. Lainé ${ }^{1,2}$ \\ 1 Laboratory of Mechanics and Physics of Materials, ENSMA, Téléport2, 1 avenue Clément Ader, BP40109, \\ F-86961 Futuroscope Chasseneuil - France \\ 2 Apollor, 1 rue du Bois de la Sivrite, 54500 Vandoeuvre-lès-Nancy - France \\ e-mail: grandidier@lmpm.ensma.fr - laine@apollor.com
}

\begin{abstract}
Résumé - Identification par algorithme génétique d'une loi de comportement prenant en compte les effets de vitesses et de pression hydrostatique - La satisfaction des exigences des cahiers de charges passe par l'amélioration des modèles de comportement des matériaux polymères utilisés dans la conception de pièces structurales notamment dans le secteur automobile.

C'est dans ce contexte qu'Apollor, en collaboration avec de grands groupes industriels tels que Basell, Renault S.A., Renault Truck, Solvay et Visteon a mis en place une étude de recherche et développement sur la problématique du comportement des polymères subissant des grandes vitesses de déformations. La méthodologie de caractérisation adoptée s'appuie sur un outil expérimental accompagné d'outils numériques d'identification par méthodes inverses des paramètres mécaniques d'une loi de comportement à implanter dans les codes de calcul.

Ainsi, les effets de pression hydrostatique et de vitesses sur différents paramètres ont été introduits dans une loi de comportement de la littérature. Ensuite, un protocole d'identification des paramètres de la loi a été développé. Il est basé sur un algorithme génétique qui lui confère toute sa robustesse.
\end{abstract}

\footnotetext{
Abstract - Identification by Genetic Algorithm of a Constitutive Law Taking into Account the Effects of Hydrostatic Pressure and Speeds - Meeting technical specifications requires the improvement of polymeric material behaviour used in the conception of structural parts notably in the automotive sector.

It is in this context that Apollor, in collaboration with several industrial groups including Basell, Renault S.A., Renault Truck, Solvay and Visteon coordinated a research and development study on the problematic of polymer behaviour at high strain speeds.

The methodology of characterization adopted includes an experimental tool backed up by numeric tools to identify by inverse methods the mechanical parameters of a literature model used in computation software.

Thus, effects of hydrostatic pressure and speed on different parameters have been introduced in the model. Then, an identification protocol of the model parameters has been developed. It is based on a genetic algorithm that confers high efficiency.
} 


\section{INTRODUCTION}

The increasing computation power allows us to envisage the predictive approach for complex parts and structures. This is particularly advantageous, if we want to meet the demanding requirements of the technical specifications, notably in the automotive sector where it is an important need to reduce, and even to abolish costs and time related to the perfecting of prototypes.

The reliability of the predictive approach is highly dependent on the richness of the mathematical models, incorporated in the computation codes, as well as of the identification protocols (for definition of parameters) related to them. This task is even more difficult for parts made of polymeric material undergoing high solicitation rates.

It is in this context that Apollor, in collaboration with a consortium of firms (Basell, Renault S.A., Renault Truck, Solvay and Visteon) is developing an efficient methodology for characterization of polymeric materials.

This article presents an experimental and numerical protocol of identification by genetic algorithm of the parameters of a constitutive law from the literature that has been modified to take into account the effects of hydrostatic pressure and of strain rate.

Before setting out the experimental and numerical methodology, as well as the results of the tests illustrating the reliability of the process, we present succinctly the theoretical basis of the constitutive law. The second purpose of this article is to evaluate the advantages of describing the effects of hydrostatic pressure. The methodology is applied in the framework of classical laws considering the effects of hydrostatic pressure. The comparison allows to quantify improvement in model predictions. This information is very important for automotive engineers.

\section{BASIS OF THE CONSTITUTIVE LAW}

\subsection{Thermodynamic Origin}

The existence of a thermodynamic potential has been supposed, from which the state laws are derived. This potential is the Helmholtz specific free energy $\Psi$ defined by:

$$
\Psi=e-T s
$$

with $T$ the temperature, $e$ the specific internal energy and $s$ the specific entropy.

The Helmholtz specific free energy is (additively) decomposed into a reversible elastic part $\Psi_{\mathrm{e}}\left(\underline{\varepsilon}^{\mathrm{e}}, T\right)$ and a nonreversible viscoplastic part $\Psi_{\mathrm{vp}}(p, \underline{\alpha}, T)$. The elastic part depends on the elastic strain $\varepsilon^{e}$ and on temperature, whereas the viscoplastic part is sensitive to the hardening variables $p$ and $\alpha$ and to temperature.

$$
\Psi=\Psi_{\mathrm{e}}\left(\underline{\varepsilon}^{\mathrm{e}}, T\right)+\Psi_{\mathrm{vp}}(p, \underline{\alpha}, T)
$$

Application of the Clausius-Duhem inequality allows us to obtain the relations (3) below:

$$
\underline{\sigma}=\rho \frac{\partial \Psi}{\partial \underline{\varepsilon}} ; \quad s=-\rho \frac{\partial \Psi}{\partial T} ; \quad \underline{X}=\rho \frac{\partial \Psi}{\partial \underline{\alpha}} ; \quad R=\rho \frac{\partial \Psi}{\partial p}
$$

with $\rho$ the volumic mass of the body, and $\underline{X}$ and $R$ the respective dual variables of the hardening variables $p$ and $\underline{\alpha}$.

The kinematic hardening (4) suggested by Chaboche and Lemaitre (1985) and by Chaboche (1990) has a linear term and a nonlinear term being indicative of the evanescent memory effect of the strain path:

$$
\underline{\dot{X}}=\frac{2}{3} C \underline{\dot{\varepsilon}}^{\cdot v p}-\gamma \underline{X} \dot{p}
$$

where $\dot{p}$ is the tensor modulus of cumulated viscoplastic strain rates. $C$ and $\gamma$ are both constants of the material. This hypothesis is treated with caution for polymers but it remains admissible as starting point.

In order to reproduce a nonlinear hardening effect versus strain and without restoration effects with elapsed time, the authors have suggested the following form of the thermodynamic potential $\Omega$.

$$
\Omega=\Omega\left(J_{2}(\underline{\sigma}-\underline{X})-k+\frac{\gamma}{2 C} J_{2}^{2}(\underline{X})-\frac{2 \gamma C}{9} J_{2}^{2}(\underline{\alpha}) ; T\right)
$$

$k$ being another constant of the material and $J_{2}$ the second invariant of the considered tensor. The constants $k, C$ and $\gamma$ may be as a function of temperature.

This particular potential is a priori different from the yield surface, consequently the model comes within the general framework of the non associated plasticity.

\subsection{Thermodynamic Formulation for Polymeric Materials}

The idea of superposition of two forms (linear and nonlinear) of kinematic hardening mechanism adopted by Chaboche and Lemaitre (1985) has been taken up by Chambaudet (2001) for the thermodynamic formulation of thermoplastic and thermosetting polymers. He then expresses the Helmholtz free energy through the relation below:

$$
\rho \Psi=\rho \Psi^{\mathrm{e}}+\rho \Psi^{\mathrm{vp}}=\rho \Psi^{\mathrm{e}}+\frac{1}{3} C \underline{\alpha}: \underline{\alpha}+\mathrm{h}(p)
$$

where $\mathrm{h}(p)$ is a function defined by:

$$
\begin{aligned}
\mathrm{h}(p)=R_{0} p+ & {\left[Q_{1} p-\frac{Q_{1}}{B_{1}}\left(1-\mathrm{e}^{-B_{1} p}\right)\right] } \\
& +\left[Q_{2} p-\frac{Q_{2}}{B_{2}}\left(1-\mathrm{e}^{-B_{2} p}\right)\right]
\end{aligned}
$$

with $R_{0}$ the initial elasticity threshold and $Q_{1}, Q_{2}, B_{1}$ and $B_{2}$ new coefficients of the material. The application of the 
Equations (3) enables the following expressions of $R$ and $\underline{X}$ to be derived:

$$
R=R_{0}+Q_{1}\left(1-\mathrm{e}^{-B_{1} p}\right)+Q_{2}\left(1-\mathrm{e}^{-B_{2} p}\right), \quad \underline{X}=\frac{2}{3} C \underline{\alpha}
$$

To describe the evolution of the internal variables, the dissipation potential $\varphi^{*}$, dual of the previous one is determined (Halphen and Nguyen, 1975). Restoring effects are here neglected by identifying them with the viscoplastic dissipation potential:

$$
\varphi^{*}=\Omega_{\mathrm{vp}}
$$

If $\underline{\sigma}^{\prime}$ and $\underline{X}^{\prime}$ are the tensor deviators of same names and $\underline{\mathbf{n}}$ the tensor of the normal to the load surface, the state laws can be written as follows:

$$
\begin{gathered}
\underline{\dot{\varepsilon}}=\rho \frac{\partial \varphi^{*}}{\partial \underline{\sigma}}=\rho \frac{\partial \Omega_{\mathrm{vp}}}{\partial \underline{\sigma}}=\frac{3}{2} \frac{\partial \Omega_{\mathrm{vp}}}{\partial J_{2}} \frac{\underline{\sigma}^{\prime}-\underline{X}^{\prime}}{J_{2}(\underline{\sigma}-\underline{X})}=\dot{p} \underline{\mathbf{n}} \\
\left.\dot{\mathrm{p}}=\frac{\partial \Omega_{\mathrm{vp}}}{\partial \mathrm{R}}=\frac{\partial \Omega_{\mathrm{vp}}}{\partial J_{2}}=\left(\frac{2}{3} \dot{\underline{\varepsilon}}: \underline{\mathrm{vp}}\right)^{\cdot \mathrm{vp}}\right)^{1 / 2} \\
\underline{\dot{\alpha}}=\underline{\dot{\varepsilon}}-\frac{3}{2 C} \gamma \underline{\mathrm{X}} \dot{p}
\end{gathered}
$$

\subsubsection{Hydrostatic Pressure Effect and Sensitivity to the Speed on the Moduli}

The hydrostatic pressure effect is introduced in the dissipation potential by modulating the first and the second invariants of stresses and hardenings via a parameter a (see for example $\mathrm{Hu}$ et al., 1996). This gives:

$$
\Omega_{\mathrm{vp}}=\frac{K}{N+1}\left(\frac{(1-\mathrm{a}) J_{2}(\underline{\sigma}-\underline{\mathrm{X}})-R+\operatorname{atr}(\underline{\sigma})}{K}\right)^{N+1}
$$

with $K$ and $N$ the Norton-Hoff law coefficients for the influence on strain rates. $\underline{I}$ being the identity tensor, the expression of the tensor of the external normal to the load surface takes then the following form:

$$
\underline{\mathbf{n}}=(1-a) \frac{3}{2} \frac{\underline{\sigma}^{\prime}-\underline{\mathrm{X}}^{\prime}}{J_{2}(\underline{\sigma}-\underline{\mathrm{X}})}+a \underline{\mathbf{I}}
$$

\subsubsection{Kinematic Hardenings}

In order to dissociate the effects of the structural hardening from the viscoplastic flow, Chaboche (1995) and Chambaudet (2001) decompose additively the hardening term $\underline{X}$ in two terms $\underline{X}_{1}$ (structural hradening) and $\underline{X}_{2}$ (plastic flow).

Whereas the term $\underline{X}_{2}$ keeps the same form as the initial term $\underline{X}$, in order to assure the evanescent memory of the strain path, the term $\underline{X}_{1}$ governs the structural hardening at high strains. Their expressions are given below:

$$
\underline{\mathrm{X}}_{1}=\frac{2}{3} C_{1} \underline{\varepsilon}^{\mathrm{v} p} \quad \underline{X}_{2}=\frac{2}{3} C_{2} \underline{\varepsilon}^{\text {vp }}-G_{2} \underline{X}_{2} \dot{p}
$$

These formulations enable us to substitute the initial material coefficients $C$ and $G$ for the $C_{1}, C_{2}$ and $G_{2}$ coefficients.

\section{METHODOLOGY FOR IDENTIFYING THE PARAMETERS OF THE CONSTITUTIVE LAW}

\subsection{Experimental Protocol}

The benchtests of Videotraction and Videoshear by Apollor enable contact-free measurements of the true values (true stresses and true strains). They also enable tests to be performed at regulated true strain rates $\left(G^{\prime}\right.$ Sell and Jonas, 1979)

In order to "cover" a wide range of strain rates, uniaxial tension tests have been performed (Apollor) with crosshead speeds from 1 to $100 \mathrm{~mm} / \mathrm{min}$. For important tensile speeds ( 600 and $6000 \mathrm{~mm} / \mathrm{min}$ ), the data have been provided by the firm Basell.

The geometry of the uniaxial tension test specimens is defined by the ASDM1822 standard modified (Fig. 1). Concerning the shear test specimens, this is the one used by Apollor.

For each uniaxial tension test, the measurements of longitudinal and transversal strains are performed. Concerning the shear tests, the glides in the center of the shear groove are measured. The main tests carried out are shown in Figure 2.

The uniaxial tension and shear tests enabled an experimental database to be built, which was necessary for a reliable identification of the parameters of the constitutive law by genetic algorithm that we present briefly below.

\subsection{Numerical Protocol - Genetic Algorithm Principle}

The literature has an abundance of references to the numerical methods for iterative optimization used for parameter identification. The most prominent methods are the gradient methods and one of the strongest is known as the Levenberg-Marquardt method. However, they require derivations (frequently a source of numerical noise) and they depend strongly on the initial values of the parameters to be determined. Moreover, when there is a great number

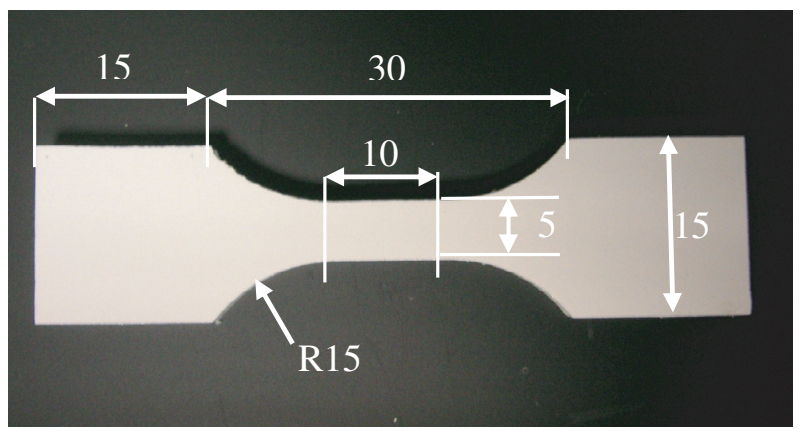

Figure 1

Modifed uniaxial tension test specimens. 


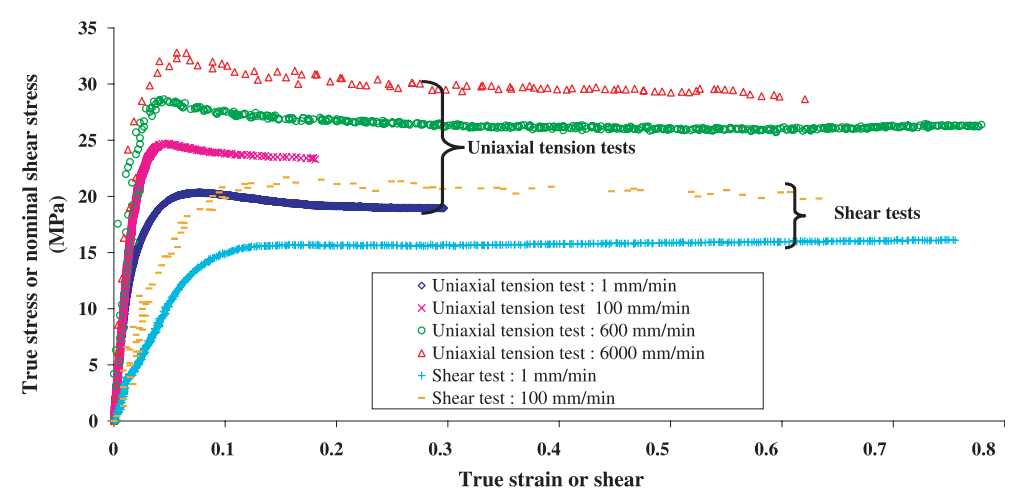

Figure 2

Tests performed for identification on the unfilled PP.

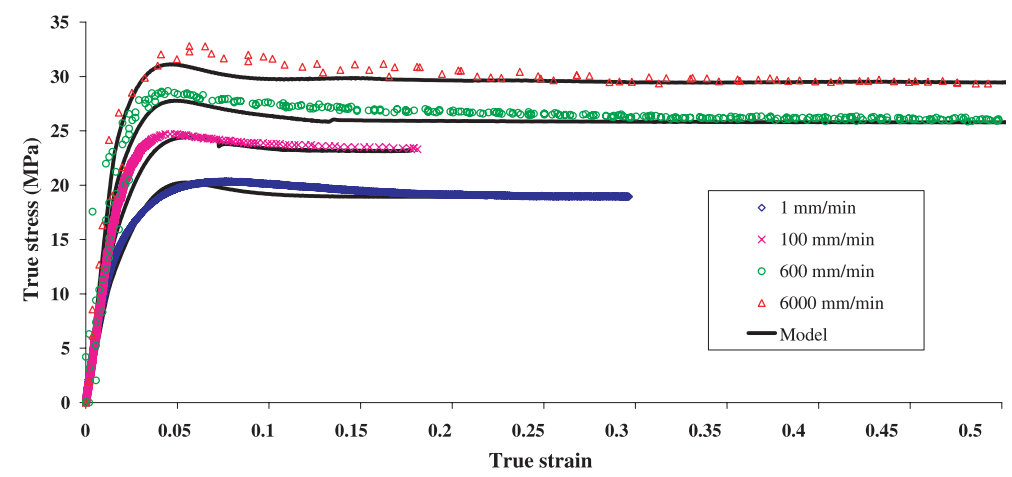

Figure 3

Identification of the parameters with four uniaxial tension curves on the PP.

of parameters, (as in the case that interests us), there is an important risk of "coming" at local minima.

Thus, genetic algorithms are increasingly being adopted. These appeared a few decades ago, they are based on a "random" examination of a combination of parameters values taken in the acceptable intervals. The genetic algorithm (Koza, 1992) is a generational algorithm with elitism of the best individual, fitness proportionate selection scheme, double crossover and mutation operators. The power of present day computers gives the possibility to manage a large population of "individuals" being able to evolve according to the evaluation criteria, reproduction and mutation probabilities, until they give a good solution. Our approach involves the use of arrays of real-valued numbers instead of bit strings to represent chromosomes. The behaviour parameters or chromosomes are chosen randomly between prescribed limits. The population size contains about fifty possible solutions. In order to evaluate each member of the population, experimental curves are simulated with parameters. Efficiency corresponds to the sum of the gap between experimental points and numerical prediction points. The existing popu- lation is selected randomly to breed a new generation. Then fitness is assigned to possible solutions (i.e. chromosomes) by the fitness function (power function). This fitness level is used to associate a probability of selection to each individual chromosome. The selected individuals are paired randomly to procreate and crossover is carried out with a crossover probability (0.9) defined by the user. Next step is to generate a second generation population of solutions from those selected. Two new solutions are created with two points crossover technique applied on real-valued numbers. The double point crossover operator uses two randomly points on chromosomes in order to mix the genes of both parents. The mutation operator necessitates the definition of the probability that an arbitrary chromosome (one parameter) in a individual may be changed from its original state. This probability is defined by user $(0.25)$. The algorithm stops when the maximum number (2000) of generation is achieved.

The genetic algorithms have the merit of excluding the difficulties related to derivation and initialization. It is easy to incorporate into them disturbance functions (mutation) 


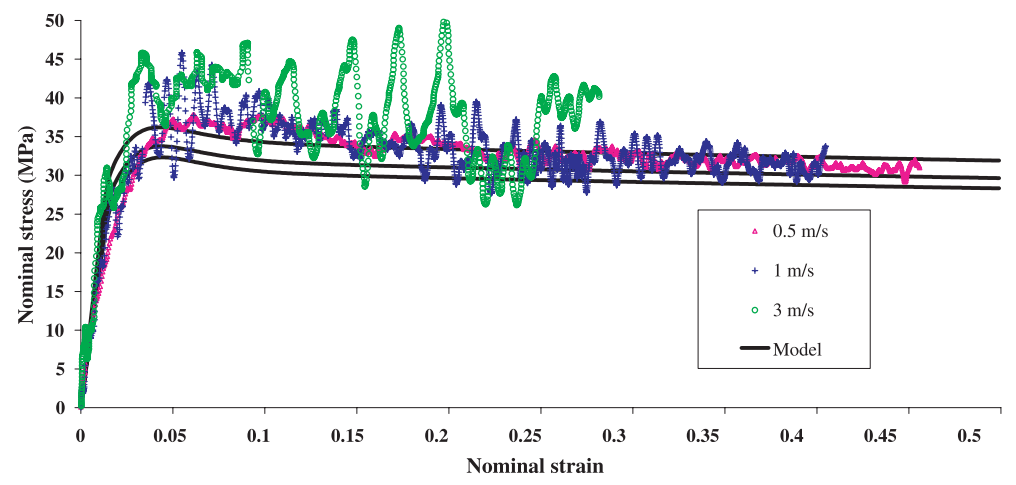

Figure 4

High speed behaviour extrapolation from data at low rate.

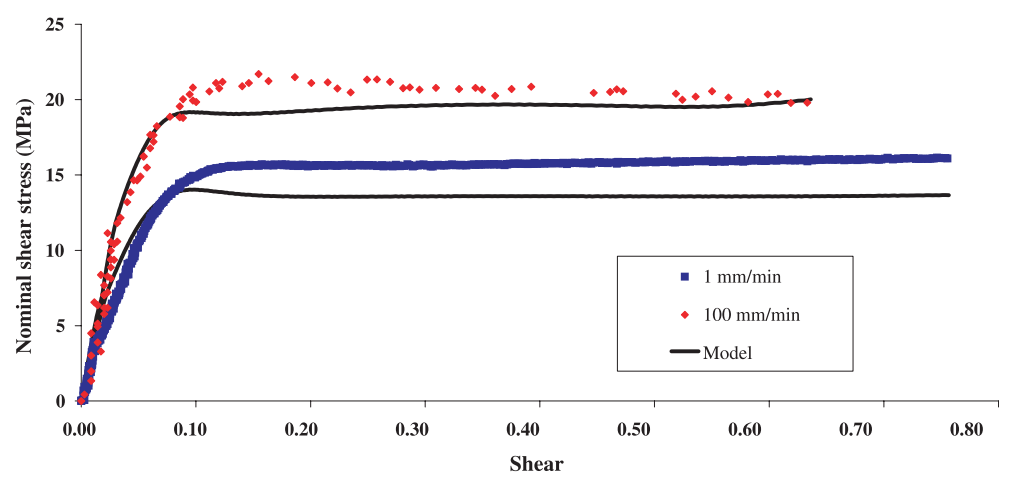

Figure 5

Shear behaviour extrapolation from uniaxial tension data.

able to rapidly locate good solutions, even for difficult search spaces.

This method has been finally elaborated for the identification of the law parameters, with the results presented below.

\section{RESULTS}

In order to underline the effects of speed and hydrostatic pressure taken into account in the constitutive law, three procedures of identification have been run:

- In the first one, four uniaxial tension test curves at 1, 100, 600 and $6000 \mathrm{~mm} / \mathrm{min}$ are used.

- In the second one, the same test curves are used without taking into account the hydrostatic pressure parameter.

- In the last procedure, a shear curve completes the four uniaxial tension test curves.

In each of these three procedures, an extrapolation to high tensile speeds, as well as for shear, enables us to estimate the quality of the model.

\subsection{Identification with Four Uniaxial Tension Curves}

Test/theoretical model concordances (Fig. 3) indicate the quality of the identification performed. Due the speed effect being taken into account, it is possible to extrapolate the behaviour at high solicitation rates, as shown in Figure 4. Shear extrapolation (Fig. 5), is possible, by taking into account the hydrostatic pressure effect. For these extrapolations, we note maximum differences of around $10 \%$ between test and model.

\subsection{Underlining of the Hydrostatic Pressure Effect in the Constitutive Law}

As indicated in Figure 6, the results show that the identification without the pressure effect leads to "acceptable" behaviours in the interval 1 to $6000 \mathrm{~mm} / \mathrm{min}$ (however less than in the previous case). On the other hand, we notice that the model without pressure effect does not enable to extrapolate correctly the shear (Fig. 7) nor high tensile speeds (Fig. 8). This underlines clearly the interest of taking into account the hydrostatic pressure effect in the model. 


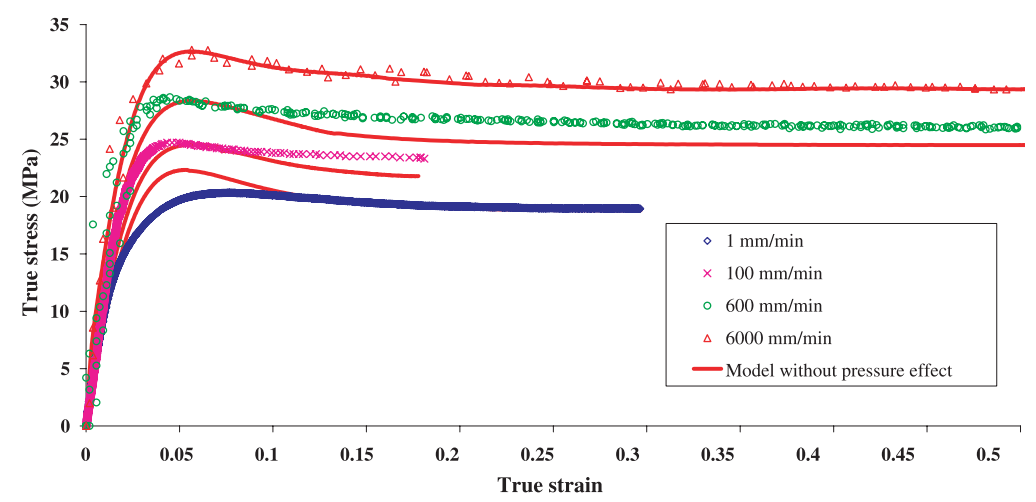

Figure 6

Underlining of the hydrostatic pressure effect of the constitutive law.

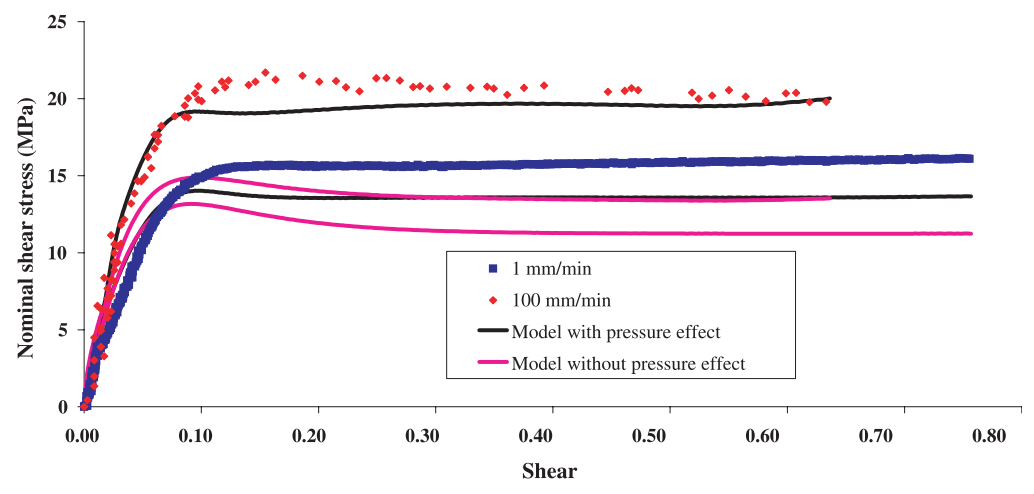

Figure 7

Underlining of the pressure effect in the shear extrapolation.

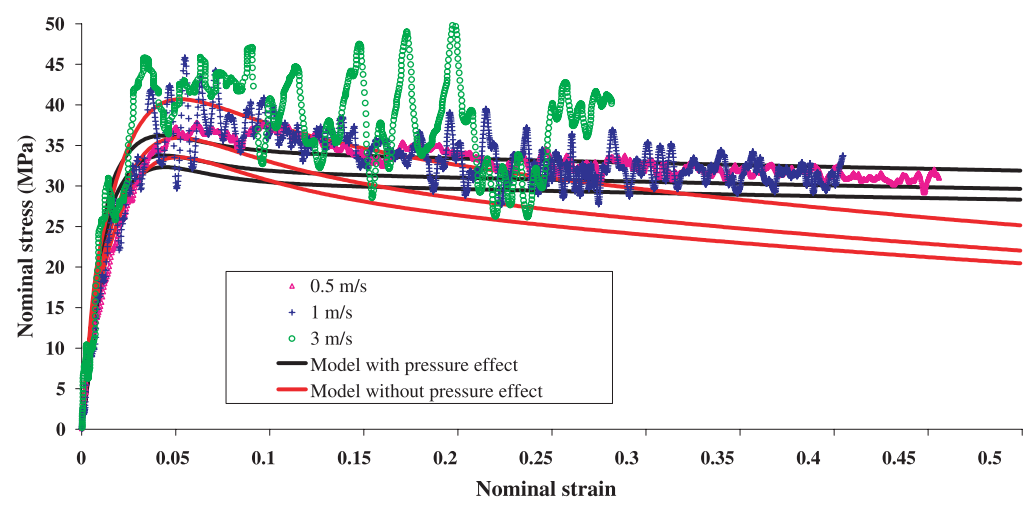

Figure 8

Underlining of the pressure effect at high tensile speeds.

\subsection{Identification with Four Uniaxial Tension Curves and One Shear Curve}

The use of a shear curve with the four previous uniaxial tension curves enables to improve the behaviour only at low stress and shear strains levels (Fig. 9). However, at more important stresses $(>10 \%)$, the effect of taking into account of the shear curve in the identification is not very visible on the uniaxial tension tests curves used. However, it has to be noted that in this identification, the uniaxial tension 


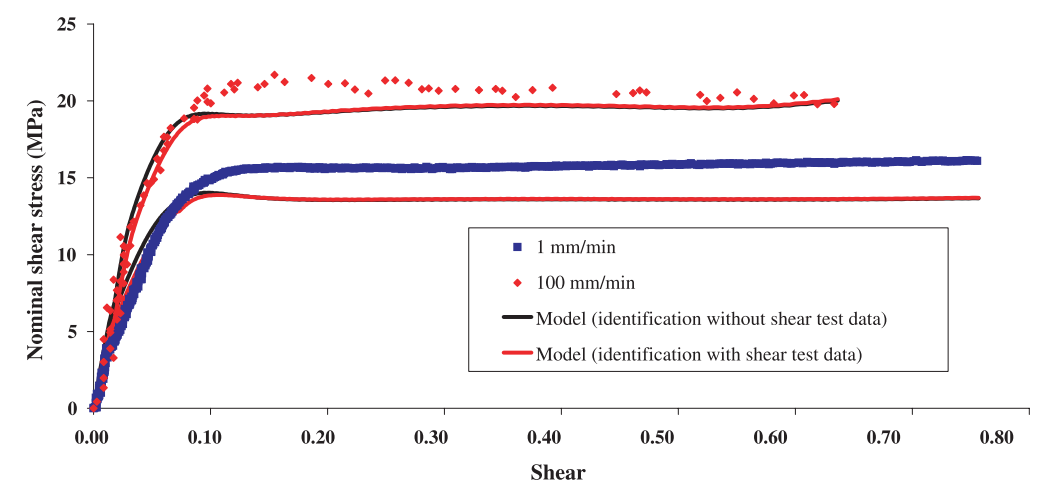

Figure 9

Effect of taking into account of the shear in the identification.

"weight", (four curves) is predominant over of those of the shear (a curve). So, by coupling four uniaxial tension curves with four shear curves, we can expect to be able to account more closely for the speed dependance of the two solicitation modes.

\section{CONCLUSION}

The viscoplastic constitutive law presented here takes rigorously into account the strain rate effect and that of the hydrostatic pressure which are very important for the behaviour of thermoplastic polymers at high deformation rates.

The methodology of identification of the parameters by genetic algorithms has the merit of not requiring any numerical derivation (source of error) and particularly to be free from the difficulties of initialization, compared to the standard iterative methods. It has allowed reliable estimation of the parameters in a minimal time, taking into account the hydrostatic pressure and the modulus, as well as the other parameters of the constitutive law.

The robustness of this process is illustrated by the results of identification and extrapolation at high speed and with a shear strain.

\section{REFERENCES}

1 Chaboche, J.-L. and Lemaitre, J. (1985) Mécanique des matériaux solides, Dunod, Paris. Édition anglaise (1992) Cambridge University Press.

2 Chaboche, J.-L. (1990) Constitutive equations for cyclic plasticity and cyclic viscoplasticity, Int. J. Plasticity, 5, 247-302.

3 Chambaudet, S. (2001) Étude et modélisation du comportement mécanique de la matrice polymère dans un composite à fibres de carbone sous compression transverse, Thesis, Université INPL, École des Mines de Nancy.

4 Halphen, B. and Nguyen, Q.S. (1975) Sur les matériaux standard généralisés, J. Mécanique, 14.

$5 \mathrm{Hu}$, G.K., Schmidt, F., Baptiste, F. and François, D. (1996) Viscoplastic analysis of adhesive joints, J. Appl. Mech., 36, 21-26.

6 Chaboche, J.-L. (1995) Formalisme général des lois de comportement applications aux métaux et polymères, Introduction à la mécanique des polymères, INPL, Nancy, 119-140.

7 G'Sell, C. and Jonas, J-J. (1979) Determination of the plastic behavior of solid polymers at constant strain rate, J. Mater. Sci., 14, 583-591.

8 Koza, J. (1992), Genetic Programming: On the Programming of Computers by Means of Natural Selection, MIT Press.

Final manuscript received in September 2006 